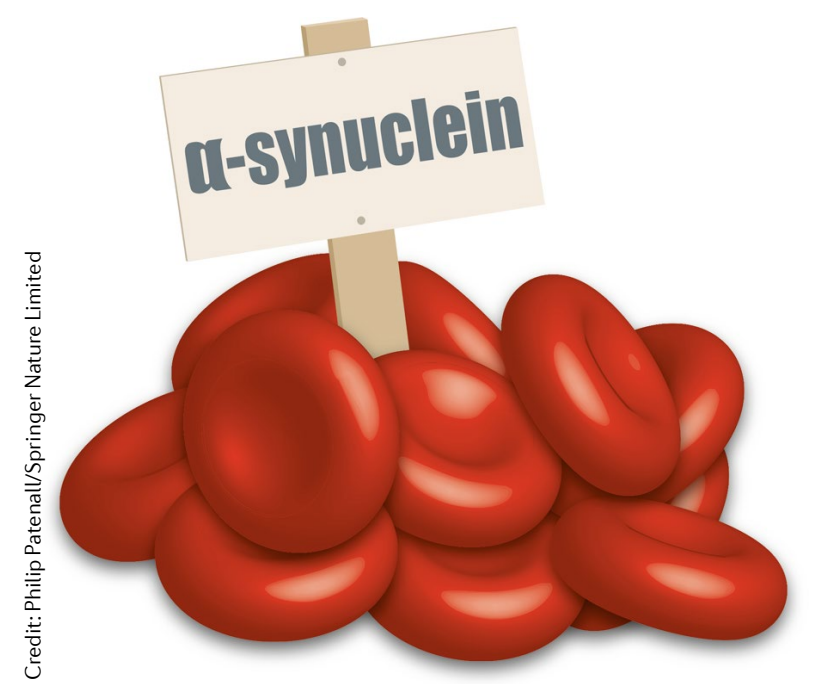

"These results add to recent reports of peripheral $\alpha$-synuclein levels as a potential biomarker of cognitive impairment in PD," says Ng. "However, longitudinal cohorts involving a larger number of patients with PD of varying disease stages as well as age-

\section{overall levels} of $\alpha$-synuclein were significantly higher among patients with PD matched and sex-matched healthy controls are required to validate these results."

Ian Fyfe synuclein detected by single molecule array is increased in PD. Ann. Clin. Transl Neurol. https:// doi.org/10.1002/acn3.729 (2019)

basis of their reactivity to full-length a-synuclein.

Using an in vitro assay, Pascual and colleagues showed that the three antibodies inhibited the seeding of intracellular $\alpha$-synuclein aggregation. The antibodies also recognized Lewy pathology in post-mortem brain tissue from patients with PD.

"Our findings suggest that the memory B cell compartment of patients with PD is a potential source

the three antibodies inhibited the seeding of intracellular $\alpha$-synuclein aggregation to $a$-synuclein, which could have therapeutic and biomarker applications," concludes Pascual. "Future studies will provide a better understanding of the extent of $a$-synuclein specificities that exist in the natural repertoire of patients with PD and establish whether such specificities are also present in the general population."
ORIGINAL ARTICLE Ng, A. S. et al. Plasma alpha-

\section{Fibrinogen links vascular pathology to cognitive decline}

Vascular damage is frequently observed in the brains of individuals with Alzheimer disease (AD), but its relationship with amyloid- $\beta(A \beta)$ pathology, neurodegeneration and cognitive impairment is not fully understood. In a new study reported in Neuron, Katerina Akassoglou and colleagues show that cerebrovascular damage leads to deposition of the blood protein fibrinogen, which causes cognitive deficits through elimination of dendritic spines.

"Our research focuses on understanding how blood leaks in the CNS affect nervous system functions," explains Akassoglou. "We were intrigued by the strong clinical correlations between vascular pathology and cognitive decline and became interested in deciphering the mechanisms that link this pathology with neuronal dysfunction."

Dendritic spines are neuronal protrusions that receive synaptic input and are crucial for learning and memory. Using longitudinal two-photon imaging in the $5 \times F A D$ mouse model of $A D$, the investigators observed a loss of spines in the vicinity of fibrinogen deposits in the brain. Intriguingly, the fibrinogen deposits did not always colocalize with $A \beta$ plaques, suggesting that the effects of fibrinogen on dendritic spines were not dependent on $\mathrm{A} \beta$ deposition.

Through a technique known as iDISCO (immunolabelling-enabled 3D imaging of solvent-cleared organs), the researchers visualized fibrinogen, $A \beta$ and blood vessels in brain tissue from mice and patients with $A D$. These experiments confirmed the presence of focal fibrinogen deposition and blood-brain barrier (BBB) leakage, even in areas without $A \beta$ pathology. "To our knowledge, this is the first 3D imaging of BBB disruption in the solvent-cleared brain," comments Akassoglou.

Previous studies have shown that fibrinogen activates microglia by binding to the $\mathrm{CD} 11 \mathrm{~b}$ receptor, resulting in the generation of neurotoxic reactive oxygen species (ROS). Akassoglou and colleagues found that dendritic spine loss could be prevented by inhibiting ROS release. In addition, genetic disruption of the $\mathrm{CD} 11 \mathrm{~b}$ receptor binding site on the fibrinogen molecule reduced neurodegeneration and improved cognitive performance in $5 \times$ FAD mice.

"The finding that fibrinogen deposition induces pathogenic microglial activation, leading to ROS release and dendritic spine loss, might open up a new pathway for therapeutic intervention to suppress vascular-driven neurodegeneration," concludes Akassoglou. "In addition, fibrinogen and fibrin could be useful biomarkers for sensitive detection of vascular abnormalities in neurodegenerative diseases."

Heather Wood

Heather Wood

ORIGINAL ARTICLE Li, X. et al. Naturally occurring antibodies isolated from PD patients inhibit synuclein seeding in vitro and recognize Lewy pathology. Acta Neuropathol. https://doi. org/10.1007/s00401-019-01974-5 (2019) FURTHER READING Goedert, M. et al. 100 years of Lewy pathology. Nat. Rev. Neurol. 9, 13-24 (2013)
ORIGINAL ARTICLE Merlini, M. et al. Fibrinogen induces microglia-mediated spine elimination and cognitive impairment in an Alzheimer's disease model. Neuron https://doi.org/10.1016/j.neuron.2019.01.014 (2019)

FURTHER READING Petersen, M. A. et al. Fibrinogen in neurological diseases: mechanisms, imaging and therapeutics. Nat. Rev. Neurosci. 19, 283-301 (2018) | Ryu, J. K. et al. Fibrin-targeting immunotherapy protects against neuroinflammation and neurodegeneration. Nat. Immunol. 19, 1212-1223 (2018)
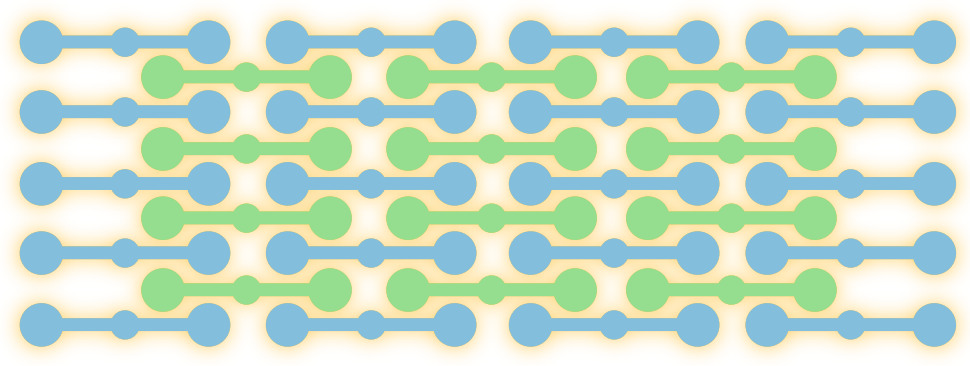

Credit: Philip Patenall/Springer Nature Limited 